Revue internationale P.M.E.

Économie et gestion de la petite et moyenne entreprise

\title{
Essai de définition et fonctionnalité de l'entreprise familiale dans une perspective africaine
}

\section{Jean-Christophe Boungou Bazika}

Volume 18, numéro 3-4, 2005

URI : https://id.erudit.org/iderudit/1008480ar

DOI : https://doi.org/10.7202/1008480ar

Aller au sommaire du numéro

Éditeur(s)

Presses de l'Université du Québec

ISSN

0776-5436 (imprimé)

1918-9699 (numérique)

Découvrir la revue

Citer cet article

Boungou Bazika, J.-C. (2005). Essai de définition et fonctionnalité de l'entreprise familiale dans une perspective africaine. Revue internationale P.M.E., 18(3-4),

11-30. https://doi.org/10.7202/1008480ar
Résumé de l'article

L'entreprise familiale n'est pas facile à définir, surtout en partant des réalités africaines. L'objectif du présent article est de souligner les difficultés méthodologiques qu'une telle définition soulève et d'indiquer les fonctions importantes que remplit l'entreprise familiale africaine. La méthodologie utilisée est basée sur une approche comparative Afrique / Occident, entreprise familiale / entreprise individuelle. L'analyse conclut que l'individualisation croissante de la société en Afrique tend à limiter la création d'entreprises familiales au profit d'entreprises individuelles. 


\title{
Essai de définition et fonctionnalité de l'entreprise familiale dans une perspective africaine
}

\author{
Jean-Christophe BOUNGOU BAZIKA
}

CERAPE, Faculté des sciences économiques, Université Marien-Ngouabi

\section{MOTS CLÉS}

\section{Entreprise familiale - Entreprise individuelle Perspective africaine - Fonctions}

\begin{abstract}
RÉSUMÉ
L'entreprise familiale n'est pas facile à définir, surtout en partant des réalités africaines. L'objectif du présent article est de souligner les difficultés méthodologiques qu'une telle définition soulève et d'indiquer les fonctions importantes que remplit l'entreprise familiale africaine. La méthodologie utilisée est basée sur une approche comparative Afrique/Occident, entreprise familiale/entreprise individuelle. L'analyse conclut que l'individualisation croissante de la société en Afrique tend à limiter la création d'entreprises familiales au profit d'entreprises individuelles.
\end{abstract}

\section{ABSTRACT}

Defining the family enterprise is no easy matter especially in the African context This article aims to underline the methodological difficulties that such definition generates and to indicate the important functions that African familial enterprise fulfils. The methodological approach used for the sake of this article is based on

\section{L'AUTEUR}

JEAN-ChRISTOPHe Boungou BAZIKA est enseignant permanent à la Faculté des sciences économiques de Brazzaville (Congo) et directeur du Centre d'études et de recherche sur les analyses et politiques économiques (CERAPE). Il a réalisé plusieurs travaux de recherche et publications sur les questions relatives à l'entrepreneuriat dans les pays africains. Adresse: Centre d'études et de recherche sur les analyses et politiques économiques (CERAPE), Faculté des sciences économiques, Université Marien-Ngouabi. Courriel: <boungoubazik@yahoo.fr>.

1. L'auteur remercie le Dr Bethuel Makosso ainsi que l'évaluateur 2 dont les remarques pertinentes ont permis d'améliorer le manuscrit. Cependant, il porte seul la responsabilité des analyses contenues dans cet article.

(C) 2005 - Presses de l'Université du Québec

Édifice Le Delta I, 2875, boul. Laurier, bureau 450, Sainte-Foy, Québec G1V 2M2 - Tél.: (418) 657-4399 - www.puq.ca

Tiré de: Revue internationale P.M.E., vol. 18, nos $3-4$, sous la direction de Louis Raymond - PME1803N

Tous droits de reproduction, de traduction et d'adaptation réservés 
African versus Western, family enterprise versus individual enterprise comparative method. The conclusion is that the increasing individualization of the African society tends to hinder the creation of family enterprises to the benefit of individual ones.

\section{RESUMEN}

La empresa familiar no es fácil de definir, sobre todo yendo de las realidades africanas. El objetivo del presente artículo consiste en destacar las dificultades metodológicas que tal definición levanta y de indicar las funciones importantes que ejerce la empresa familiar africana. Metodología utilizada se basa en un enfoque comparativo África/Occidente, empresa familiar/emprendida individual. El análisis concluye que la individualización creciente de la sociedad en África tiende a limitar la creación de empresas familiares en favor de empresas individuales.

\section{ZUSAMMENFASSUNG}

Ein Familienunternehmen zu umschreiben und zu definieren ist nicht einfach, umso mehr, wenn es sich um Unternehmen aus Afrika handelt. Die Zielsetzung des vorliegenden Artikels besteht einerseits darin, die methodologischen Schwierigkeiten zu unterstreichen, die ein solcher Definitionsversuch mit sich bringt. Andererseits sollen die wichtigen Funktionen der afrikanischen Familienunternehmen hervorgehoben werden. Das hierbei gewählte methodologische Vorgehen basiert auf dem Vergleich Afrika/westliche Hemisphäre sowie Familienunternehmen/Einzel-unternehmung. Die Schlussfolgerungen zeigen auf, dass die zunehmende Individualisierung der afrikanischen Gesellschaft, die Entstehung von Familienunternehmen zu Gunsten von Einzelunternehmungen einschränkt.

\section{Introduction}

L'entreprise familiale peut être considérée comme la première forme d'organisation hiérarchisée de la production des biens et services. Elle est née historiquement à partir de l'apparition de la propriété privée. Cela signifie que la société basée sur la propriété communautaire primitive ne pouvait connaître l'entreprise familiale, c'est-à-dire une forme de propriété distincte de la propriété collective et appartenant exclusivement à un petit groupe de personnes liées par des liens de sang ou de mariage.

Il est possible d'affirmer que l'entreprise familiale représente le fondement de l'entreprise moderne. Ce fondement comporte deux caractéristiques essentielles:

- d'un point de vue historique: la société traditionnelle que décrit de façon remarquable le Pr Penouil (1979) est l'ancêtre de la société moderne. Si la seconde est basée sur l'individu, la première trouve son fondement dans la famille au sens large du terme. Ainsi, l'organisation de la production dans la première forme de société est d'abord une organisation à caractère 
familial. Celle-ci s'est adaptée dans la société moderne, a connu quelques mutations et a même produit de grandes organisations multinationales tout en conservant le caractère familial. C'est le cas de firmes comme Michelin, Peugeot, Toyota ou Philips.

- d'un point de vue naturel: l'entreprise familiale est au cœur de l'organisation économique parce que l'entrepreneur est un preneur de risques intégré à une cellule familiale.

Comme le définissent Guitton et Vitry (1991), «l'entrepreneur est une personne (entreprise individuelle) ou un groupement de personnes (entreprise sociétaire) qui supporte les risques de l'affaire» (p. 263). Or, assumer de tels risques avec d'autres personnes suppose l'existence préalable de relations de confiance. La famille constitue le premier creuset où se développent entre des individus des relations de confiance: confiance entre le mari et la femme, confiance entre les frères, sœurs, cousins, etc.

La justification de se pencher sur l'entreprise se rattache au débat soulevé par les mutations actuelles de la petite et moyenne entreprise. On estime que peu de travaux existent et permettent d'avoir une connaissance approfondie de l'entreprise familiale, qui constitue bien souvent la forme dominante des PME dans le monde'2.

Dans les pays africains caractérisés par la coexistence de la société traditionnelle et la société moderne, l'entreprise familiale occupe encore une place relativement plus importante que dans les sociétés capitalistes développées marquées par la désagrégation de la cellule familiale; celle-ci est de plus en plus réduite à la cellule mononucléaire.

L'objectif de cet article est d'essayer de donner une définition cohérente de l'entreprise familiale et d'en préciser les fonctions, et ce, dans une perspective africaine.

L'analyse de l'entreprise familiale sera faite en adoptant une approche comparative de l'Occident et de l'Afrique, d'une part, de l'entreprise familiale et individuelle, d'autre part, afin d'en souligner les spécificités. L'hypothèse de départ est que l'entreprise familiale assume d'importantes fonctions économiques dans les pays africains, mais qu'elle tend à être remplacée graduellement par l'entreprise individuelle à cause de l'urbanisation et de l'individualisation croissante de la société. Son importance relative découle du fait que la famille continue de constituer

2. Au congrès de l'Association internationale de recherche sur les PME (AIRPME), tenu à Montréal (Canada) en 2002, un des problèmes soulevés par les débats a été la nécessité de mener des études sur l'entreprise familiale, type d'entreprise méconnue de la communauté des chercheurs.

Revue internationale P.M.E., vol. 18, nºs 3-4, 2005

(C) 2005 - Presses de l'Université du Québec

Édifice Le Delta I, 2875, boul. Laurier, bureau 450, Sainte-Foy, Québec G1V 2M2 • Tél.: (418) 657-4399 - www.puq.ca

Tiré de: Revue internationale P.M.E., vol. 18 , nos $3-4$, sous la direction de Louis Raymond $\cdot$ PME1803N

Tous droits de reproduction, de traduction et d'adaptation réservés 
un rempart pour la sécurité des individus face à la vulnérabilité de l'environnement économique et social, même si, comme le pense Bauman (2003), les mutuelles de micro-assurance tendent à prendre de l'ampleur en raison des difficultés croissantes éprouvées par la cellule familiale. L'analyse de l'entreprise familiale exige de recourir à l'identification des modèles familiaux et de leur dynamique en Afrique. C'est pourquoi, au-delà de l'économie de l'entreprise, des travaux entrepris par des démographes, sociologues et anthropologues sur la famille serviront de référence pour appuyer notre hypothèse de départ (notamment Marie, 1997; Wakam, 1997; Vimard et N'Cho, 1997).

Notre démarche consistera d'abord à présenter une définition de l'entreprise familiale, chose difficile étant donné les contraintes méthodologiques que comporte cette définition. Ensuite seront précisées les différentes fonctions économiques de l'entreprise familiale dans une perspective africaine.

\section{Perception de l'entreprise familiale: difficultés méthodologiques et essai de définition}

Dans la définition de l'entreprise familiale apparaît une série de problèmes qu'il importe de signaler. On peut relever trois genres de difficultés: d'ordre sémantique, d'ordre limitatif et d'ordre dynamique.

\subsection{Difficulté sémantique}

L'entreprise familiale associe deux concepts: ceux d'entreprise et de famille. Il apparaît nécessaire de les définir séparément afin de mieux apprécier leur relation.

Il existe plusieurs définitions de l'entreprise et aucune ne fait l'unanimité entre les spécialistes des sciences de l'économie et de la gestion. Prenons celle de François Perroux. Pour cet auteur, l'entreprise est «une organisation de la production dans laquelle on combine les prix de divers facteurs de la production apportés par des agents distincts du propriétaire de l'entreprise en vue de vendre un bien ou des services sur le marché pour obtenir par différence entre deux prix (prix de revient et prix de vente) le plus grand gain monétaire possible» (cité dans Guitton et Vitry, 1991, p. 263). Cette définition met l'accent, entre autres, sur la séparation entre la propriété de l'entreprise et celle de certains facteurs (travail, capital, etc.). Elle met également en évidence l'objectif poursuivi, soit celui d'obtenir le profit maximal.

Deux problèmes se posent: lorsque la propriété de l'entreprise est associée à une participation directe du propriétaire au travail, avons-nous là une forme d'organisation que l'on peut appeler entreprise? Bon nombre d'entreprises individuelles implantées dans les pays africains où le patron travaille avec ses employés et

Revue internationale P.M.E., vol. 18, n ${ }^{\text {os }} 3-4,2005$

(C) 2005 - Presses de l'Université du Québec

Édifice Le Delta I, 2875, boul. Laurier, bureau 450, Sainte-Foy, Québec G1V 2M2 • Tél.: (418) 657-4399 - www.puq.ca

Tiré de: Revue internationale P.M.E., vol. 18, nos $3-4$, sous la direction de Louis Raymond P PME1803N

Tous droits de reproduction, de traduction et d'adaptation réservés 
apprentis, et possède certains facteurs en propre comme le bâtiment ou le terrain ne pourraient pas être définies comme des entreprises au sens «perrousien» du terme. Alors, adopter une telle définition comporte le risque méthodologique d'exclure du champ de recherche bien des entreprises africaines. En effet, celles-ci concentrent non seulement le capital de démarrage, mais aussi certaines immobilisations, une partie du travail, voire la matière première, quand il s'agit de transformation de produits agricoles, entre les mains d'une seule personne, soit le propriétaire de l'entreprise.

Le deuxième problème réside dans l'objectif fixé: le gain le plus élevé. Dans le contexte africain, Torrès (2001) montre que l'entreprise ne vise pas la croissance, mais bien la sécurité des capitaux investis. L'atteinte de cet objectif est entravé par l'étroitesse du marché découlant du faible pouvoir d'achat des ménages et de l'instabilité de l'environnement de l'entreprise (Hernandez, 2002; Hugon, 1995). Ainsi, l'entreprise est marquée par son caractère informel qui lui permet de mieux s'adapter à son milieu. Celle-ci vise l'obtention du minimum de profit, celui qui permet d'assurer la survie de l'entreprise et donc la survie du ménage puisque ce profit vise à couvrir avant tout les dépenses de consommation du propriétaire et celles de sa famille. L'objectif prioritaire n'est pas l'autofinancement de l'entreprise pour contribuer à sa croissance. Ainsi, l'objectif tel qu'il est défini par Perroux n'est pas adaptable au contexte de la petite et moyenne entreprise africaine.

Une autre définition de l'entreprise qui est largement répandue est celle élaborée par Schumpeter. Selon cet auteur, l'entreprise est un lieu où s'effectuent de nouvelles combinaisons de facteurs, de nouveaux produits, de nouveaux débouchés et de nouvelles méthodes d'organisation de la production. En un mot, l'entreprise est une organisation qui innove en permanence dans un processus de «destruction-créatrice».

Cette définition de l'entreprise, si elle peut permettre de caractériser certaines formes d'organisation de la production marquées par un haut degré d'innovation, formes que l'on désigne aujourd'hui par le concept d'entreprises «technologiques», il est difficile d'affirmer qu'une telle définition pourrait correspondre à la majorité des entreprises dans le monde, particulièrement en Afrique. Kombou (2002), analysant l'activité des PME au Cameroun, aboutit à la conclusion que celles-ci ne sont pas innovantes. Elles seraient même conservatrices, se contentant d'imiter les nouvelles technologies et les nouveaux produits conçus ailleurs, dans le monde occidental. Dans le même sens, Boussetta (2003), appréciant la manière dont les PME marocaines appréhendent l'innovation, déduit à partir d'une enquête de terrain portant sur 100 entreprises de différents secteurs, que ces entreprises innovent très peu en dépit du fait que toutes considèrent l'innovation comme une stratégie déterminante pour faire face à la concurrence des entreprises de l'Union européenne.

Revue internationale P.M.E., vol. 18, ${ }^{\text {os }} 3-4,2005$

(C) 2005 - Presses de l'Université du Québec

Édifice Le Delta I, 2875, boul. Laurier, bureau 450, Sainte-Foy, Québec G1V 2M2 • Tél.: (418) 657-4399 - www.puq.ca

Tiré de: Revue internationale P.M.E., vol. 18, nos 3-4, sous la direction de Louis Raymond • PME1803N

Tous droits de reproduction, de traduction et d'adaptation réservés 
Ainsi, si l'on adopte la définition schumpétérienne de l'entreprise, il faudrait exclure la quasi-totalité des entreprises africaines. Seule la petite minorité représentée par les filiales des firmes multinationales implantées en Afrique serait alors comprise dans la catégorie de vraies entreprises puisqu'elles font preuve d'innovation continuelle.

Enfin, lorsqu'on se réfère à ce qu'il est convenu d'appeler l'économie néoinstitutionnelle, la définition de l'entreprise est celle élaborée par Coase en $1932^{3}$. «Une relation appelée firme émerge parce qu'il est coûteux d'utiliser le mécanisme des prix.»

Ainsi, l'entreprise est définie comme une organisation qui permet à l'entrepreneur de diriger des ressources afin d'épargner certains coûts de marché. L'entreprise permet alors de faire baisser les coûts de transactions. Dans la même lignée, Willamson ${ }^{4}$, approfondissant l'analyse de Coase, montre que l'entreprise est une organisation basée sur la passation de contrats qui traduisent la rationalité limitée des acteurs, l'opportunisme et l'asymétrie d'information. La passation des contrats incorpore non seulement les coûts se rattachant à la recherche de l'information (coûts ex ante), mais aussi à la surveillance de l'exécution du contrat (coûts ex post). Le recours à des institutions extra-économiques est souvent nécessaire (tribunaux, huissiers, etc.) pour valider les contrats et régler les litiges éventuels dans leur application.

Le problème que pose cette définition est que l'entreprise africaine est une organisation basée sur la passation de contrats non écrits, non formalisés. Ainsi, les coûts de transaction sont davantage minimisés, l'entrepreneur n'ayant juridiquement aucune obligation à l'égard de l'employé. La confiance seule suffit et exprime la base de la relation entre les deux acteurs. Lorsque cette confiance est rompue du fait de la défiance d'un des acteurs, le contrat oral est alors remis en cause. Le recours aux institutions censées garantir l'exécution des contrats est plutôt rare, d'une part, parce que l'on veut préserver les relations sociales et, d'autre part, parce que l'absence de contrats écrits empêche la compétence des institutions judiciaires de s'exercer.

Par conséquent, la définition «coasienne» de l'entreprise est difficilement applicable aux PME en Afrique.

\subsection{Difficultés de champ de délimitation}

La deuxième principale difficulté tient au concept de famille. Cette notion comme celle d'entreprise n'est pas facile à cerner; elle pose des problèmes surtout d'ordre limitatif. En effet, la famille se définit comme «une unité de base qui englobe

3. Cité par Soulié, 1992.

4 Idem.

Revue internationale P.M.E., vol. 18, $\mathrm{n}^{\text {os }} 3-4,2005$

(C) 2005 - Presses de l'Université du Québec

Édifice Le Delta I, 2875, boul. Laurier, bureau 450, Sainte-Foy, Québec G1V 2M2 - Tél.: (418) 657-4399 - www.puq.ca

Tiré de: Revue internationale P.M.E., vol. 18 , nos $3-4$, sous la direction de Louis Raymond • PME1803N

Tous droits de reproduction, de traduction et d'adaptation réservés 
non seulement les descendants et alliés, mais également un ensemble de groupes satellites qui peuvent constituer une clientèle...» (Penouil, 1979). «Au sein de cette unité, affirme Penouil, s'établissent des relations de subordination, de respect, mais aussi d'entraide et de coopération» (p. 23).

Le premier problème est associé à la notion d'appartenance: qui est membre de la famille et qui ne l'est pas? Si, en Occident, la famille est constituée par le père, la mère et leurs enfants naturels ou d'adoption, à savoir deux générations, en Afrique, elle englobe plus de deux générations et intègre d'autres membres indirects. Ainsi, la famille comprend le grand-père, la grand-mère, le père, la mère, leurs enfants et les cousins de ces derniers du côté maternel (famille matriarcale) ou du côté paternel (famille patriarcale).

Une telle composition de la famille élargie pose de réels problèmes d'appartenance surtout lorsque les membres ont été dispersés et que les plus âgés n'ont aucune référence de la lignée attestant l'appartenance directe ou indirecte à la famille de tel ou tel individu. En Afrique, la mémoire demeure le seul outil pouvant confirmer ou infirmer la lignée.

Le second problème se rattache au mariage. Si, en Occident, le mariage est un facteur qui engendre une nouvelle famille distincte de la famille d'origine des deux époux, en Afrique, les liens de mariage conduisent à des alliances et à la coexistence entre deux familles: celle de l'époux et celle de l'épouse. Cette situation d'alliances peut être source d'accroissement de puissance (alliances entre familles riches qui se traduisent également par des alliances stratégiques dans les affaires) ou, au contraire, apparition de conflits aigus et parfois meurtriers. Ces conflits naissent des problèmes liés à la répartition des ressources familiales entre divers membres en nombre croissant alors que ces ressources sont limitées. Ces problèmes peuvent prendre une tournure tragique à la suite de la mort du chef de famille et de la répartition du patrimoine laissé par le défunt. Au Congo, plusieurs entreprises ont été démantelées à l'occasion du partage de l'héritage, plongeant la famille dans la pauvreté et le désarroi.

\subsection{Dynamiques des structures familiales}

Une troisième difficulté tient à l'évolution des modèles familiaux entraînant l'apparition d'une diversité de types de familles variant des formes de familles étendues traditionnelles aux formes modernes nucléaires en passant par des formes intermédiaires plus individualisées (familles monoparentales, femmes chefs de famille, familles constituées d'individus isolés, familles sans liens de consanguinité, etc.; Vimard et N'Cho, 1997).

En Côte-d'Ivoire par exemple, le groupe socioéconomique des entrepreneurs est marqué par le renforcement de l'individualisation et de la nucléarisation de la

Revue internationale P.M.E., vol. 18, ${ }^{\text {os }} 3-4,2005$

(C) 2005 - Presses de l'Université du Québec

Édifice Le Delta I, 2875, boul. Laurier, bureau 450, Sainte-Foy, Québec G1V 2M2 • Tél.: (418) 657-4399 - www.puq.ca

Tiré de: Revue internationale P.M.E., vol. 18, nos 3-4, sous la direction de Louis Raymond • PME1803N

Tous droits de reproduction, de traduction et d'adaptation réservés 
famille. C'est le ménage «restreint». À l'inverse, les salariés et cadres supérieurs sont caractérisés par une accentuation de la cohésion sociale se traduisant par l'accueil d'autres parents au sein des ménages. À l'opposé, les paysans sans terre, les commerçants et les artisans se distinguent des autres groupes socioéconomiques par une déstructuration familiale de même nature alors qu'ils exercent leur activité dans des sphères différentes (Vimard et N'Cho, 1997).

Au Cameroun, la structuration des modèles familiaux connaît une dynamique différente de celle évoquée pour la Côte-d'Ivoire. Cette différenciation concerne surtout la couche des membres de l'exécutif et des propriétaires dont font partie les entrepreneurs. Dans cette catégorie, $70 \%$ au moins des ménages sont des familles étendues, c'est-à-dire des ménages nucléaires acceptant de vivre avec d'autres membres de la famille. La nucléarisation est plus fréquente en milieu rural qu'en milieu urbain et atteint son minimum à Yaoundé pour la plupart des ménages (Wakam, 1997).

Au Togo, les mutations connues par la famille amène à distinguer cinq types: famille monoparentale, union monogame sans enfant, union monogame avec enfants, union polygame avec enfants, famille séparée avec enfants. Dans ces cinq types, c'est la famille monogame avec enfants qui domine.

Au Ghana, on observe un phénomène particulier: le resserrement de la taille des ménages (4,5 enfants contre une moyenne de 5,3 enfants en Afrique); ce resserrement autour du noyau parents-enfants se produirait aussi au détriment de l'accueil des collatéraux (Bertrand, 2003).

Ainsi, la théorie de Talcott Parsons (1955) et de William Goode $(1963)^{5}$ prédisant une généralisation du modèle familial occidental en Afrique sous l'effet de l'urbanisation et de la monétarisation doit être relativisée. Marie (1997), analysant la situation de plusieurs capitales africaines comme Dakar, Lomé et Abidjan à différentes périodes, 1960, 1980 et 1990, distingue deux types de citadins. Le premier est celui qui est né et a grandi au village et y a conservé de fortes attaches. Ces «néocitadins» de la première génération conçoivent la famille élargie comme une norme sociale intangible. Par contre, les citadins de la deuxième génération, qui sont nés et ont grandi en ville, ont peu d'attaches avec le village. Ils ont tendance à adopter le modèle familial nucléaire et à privilégier l'individu à la communauté.

En définitive, on peut affirmer que la logique qui domine est celle du compromis entre l'individualisme familial et l'accompagnement d'un minimum de devoirs communautaires en tenant compte des ressources limitées et en poursuivant des intérêts personnels (Marie, 1997).

5. Pour une présentation synthétique de ces théories, voir l'étude de Vimard et N'Cho (1997). 


\subsection{Essai de définition}

Après avoir évoqué les difficultés méthodologiques que pose la définition de l'entreprise familiale, tentons de définir ses contours et de surmonter ces difficultés.

Dans une perspective africaine, l'entreprise familiale peut être définie comme une unité chargée de produire et d'écouler sur le marché des biens et services, unité appartenant à des personnes unies par des liens de consanguinité directs ou indirects usant de contrats non formalisés dont l'objectif prioritaire est l'obtention d'un profit minimal permettant la sécurisation du capital investi et la survie des membres de la famille.

De ce point de vue, l'entreprise familiale est distincte de l'entreprise individuelle et sociétaire. S'agissant de l'entreprise individuelle, elle est distincte de l'entreprise familiale du point de vue de la propriété du capital: le capital appartient collectivement à la famille et non à un individu. Il se transmet au sein de la famille. Une autre distinction tient à la finalité de l'entreprise. Le bénéfice est réparti entre les membres de la famille par le chef de famille alors que le bénéfice de l'entreprise individuelle est accaparé par le propriétaire qui en fait un usage personnel. Le passif de l'entreprise familiale est supporté par l'ensemble des membres qui composent la cellule familiale. Cela peut aller jusqu'à engager leurs propres biens pour sauver l'entreprise ou empêcher que le gérant soit poursuivi en justice. Cette solidarité n'est pas juridique, car l'entreprise à caractère familial ne possède aucun statut juridique en tant que tel. Elle peut choisir n'importe quelle forme juridique (entreprise individuelle, SARL, société anonyme, etc.). Mais la véritable propriété du capital est entre les mains d'une famille. Le code secret qui guide ses membres est la solidarité et l'entraide, c'est un code moral ayant pour enjeux la pérennité de la famille, son honneur, sa puissance et même sa survie.

Enfin, du point de vue de sa gouvernance, l'entreprise individuelle est pilotée par le chef d'entreprise, son propriétaire qui n'accepte pas de déléguer, ne fût-ce qu'une petite parcelle de son pouvoir. Par contre, l'entreprise familiale est dirigée par un collectif de façon formelle ou informelle. Même si un individu a été chargé explicitement de diriger l'entreprise, les autres membres ont le pouvoir d'injonction sur les décisions de l'entreprise. Ce droit d'injonction constitue une force quand il limite la prise de décision arbitraire d'un seul homme. Mais il peut constituer un frein quand au sein de la famille naissent des divergences et des conflits qui paralysent la prise des décisions.

Concernant la société, la différence entre elle et l'entreprise familiale est seulement d'ordre juridique. Le statut juridique de la société permet de répartir de façon collective les droits et obligations de chaque membre. À l'inverse, lorsque l'entreprise familiale possède le statut de l'entreprise individuelle, comme c'est

Revue internationale P.M.E., vol. 18, ${ }^{\text {os }} 3-4,2005$

(C) 2005 - Presses de l'Université du Québec

Édifice Le Delta I, 2875, boul. Laurier, bureau 450, Sainte-Foy, Québec G1V 2M2 • Tél.: (418) 657-4399 - www.puq.ca

Tiré de: Revue internationale P.M.E., vol. 18, nos 3-4, sous la direction de Louis Raymond • PME1803N

Tous droits de reproduction, de traduction et d'adaptation réservés 
le cas de bon nombre d'entreprises en Afrique, la gestion et la propriété ont une apparence, mais seulement une apparence individuelle. Cependant, dans les faits, ces aspects relèvent du collectif familial qui a donné à un de ses membres seulement le mandat de le représenter et de gérer le patrimoine familial pour le compte de la famille.

\section{Différentes fonctions de l'entreprise familiale}

L'entreprise familiale assume des fonctions particulières du fait de son caractère collectif et de ses objectifs propres. Analyser la fonctionnalité de ce type d'entreprise comporte un intérêt évident: celui de mieux faire ressortir les facteurs qui différencient l'entreprise familiale des autres formes d'entreprises, en particulier de l'entreprise individuelle, et de mieux appréhender en définitive les caractéristiques distinctives de l'entreprise familiale.

Par fonction, il faut entendre les principales tâches qui sont assignées à l'entreprise dans la réalisation de son activité et la poursuite de son objectif. La littérature sur l'économie de l'entreprise distingue quelques grandes fonctions ou fonctions principales, qui constituent l'ossature même de l'entreprise, et des fonctions secondaires visant à soutenir la réalisation des fonctions principales. Les fonctions principales concernent l'approvisionnement, la production et la distribution, et les fonctions secondaires touchent l'administration, les ressources humaines, les finances et la comptabilité, et la R-D.

On peut se baser sur la définition des fonctions de l'entreprise en général pour établir celles qui sont propres à l'entreprise familiale. Il sera retenu ici quatre fonctions importantes que remplit l'entreprise familiale: la fonction administrative de répartition naturelle des tâches, la fonction technique d'incubateur des compétences, la fonction financière de financement du capital de départ, la fonction de gestion des ressources humaines à faible coût.

\subsection{Fonction administrative de répartition naturelle des tâches}

L'entreprise familiale assure une fonction dès son origine, à savoir celle de répartir les tâches selon la nature des acteurs. Les caractéristiques naturelles que nous étudierons sont le genre, l'âge et la race.

La répartition la plus évidente et ayant un caractère universel est celle basée sur le genre, entre l'homme et la femme, lorsque cette activité associe les deux conjoints. Évidemment, la nature des tâches varie en fonction de la nature de l'entreprise et du secteur économique dans lequel elle est implantée.

Revue internationale P.M.E., vol. 18, n ${ }^{\text {os }} 3-4,2005$

(C) 2005 - Presses de l'Université du Québec

Édifice Le Delta I, 2875, boul. Laurier, bureau 450, Sainte-Foy, Québec G1V 2M2 • Tél.: (418) 657-4399 - www.puq.ca

Tiré de: Revue internationale P.M.E., vol. 18, nos $3-4$, sous la direction de Louis Raymond P PME1803N

Tous droits de reproduction, de traduction et d'adaptation réservés 
Dans l'agriculture, l'entreprise familiale attribue à l'homme les travaux d'abattage des arbres, de labours et à la femme ceux relatifs au sarclage, au binage, à l'ensemencement et aux récoltes. En plus, c'est souvent elle qui est chargée de la commercialisation des produits dans le cas de la petite production marchande. Lors d'une enquête sur le secteur informel à Brazzaville (Boungou Bazika et Samba, 2002), les données et informations collectées sur 80 très petites entreprises agricoles ont montré que les producteurs de produits maraîchers répartissent l'activité de la manière suivante: l'homme se charge de produire les légumes et la femme de les écouler sur le marché. Le fruit de la vente est réparti au sein du ménage par l'homme après avoir sollicité l'avis de sa femme.

Lorsque l'entreprise est implantée dans le secteur industriel, ou dans celui des services, l'homme s'occupe de la gestion des hommes et du matériel, la femme de celle des commandes des clients ou de la comptabilité. Le principe de répartition est au fond le même que dans l'agriculture: les tâches les plus difficiles, qui prennent le plus de temps pour leur réalisation, sont confiées à l'homme, et celles qui exigent moins d'ardeur, à la femme. La logique qui sous-tend cette répartition est moins d'ordre sexiste que l'on pourrait le croire. C'est que la femme, en plus des tâches nécessaires à la bonne marche de l'entreprise, doit assumer une autre responsabilité non moins importante: celle de veiller au bon fonctionnement du ménage, à l'éducation des enfants, aux soins quotidiens nécessaires à l'équilibre du ménage. Si l'homme peut se permettre de passer toute la journée à l'entreprise, cela n'est pas possible pour la femme, à moins d'engager une personne pour l'entretien du ménage. Pour les PME africaines, une telle charge supplémentaire n'est pas envisagée si elle entraîne un déséquilibre du budget familial.

La répartition naturelle fondée sur l'âge est étroitement liée à la culture africaine qui valorise la personne en fonction de son âge. On estime que les plus âgés ont l'expérience et la sagesse et les moins âgés doivent respect et subordination à l'égard de ces aînés dont la mission sociale est de transmettre leur expérience et connaissances aux cadets à condition que ces derniers fassent preuve d'obéissance. Un proverbe congolais dit: «les oreilles ne dépassent jamais la tête». La répartition des tâches au sein de l'entreprise familiale africaine répond à cette norme sociale. Ainsi, l'aîné est chargé des tâches de conception et de direction, le cadet de celles d'exécution.

Une autre répartition naturelle des tâches peut s'appuyer sur la race lorsque l'entreprise est constituée par deux familles de races différentes ayant passé entre elles un accord de partenariat. Pour illustrer cette idée, on peut évoquer l'exemple de la société ASI regroupant un Européen et un Africain, conduisant à ce que Cornu (1998) qualifie de «management biculturel» (p. 257). L'exemple édifiant de cette entreprise spécialisée dans la production et l'exportation de la gomme arabique illustre bien cette répartition des tâches sur une base raciale. Elle a été créée par

Revue internationale P.M.E., vol. 18, nºs 3-4, 2005

(C) 2005 - Presses de l'Université du Québec

Édifice Le Delta I, 2875, boul. Laurier, bureau 450, Sainte-Foy, Québec G1V 2M2 • Tél.: (418) 657-4399 - www.puq.ca

Tiré de: Revue internationale P.M.E., vol. 18, nos $3-4$, sous la direction de Louis Raymond - PME1803N

Tous droits de reproduction, de traduction et d'adaptation réservés 
un Français et un Nigérien et symbolise l'alliance entre deux familles: la famille Wankoye (Niger) et la famille Dulon (France). «Le choix des secteurs d'intervention s'est effectué naturellement, pour partie grâce à l'alliance des compétences de deux familles» (p. 256). Ainsi, ont été utilisées dans les relations avec les fournisseurs européens les compétences du père de Dulon, ingénieur de formation. Par ailleurs, les compétences du frère de Wankoye dans le domaine commercial ont servi à établir un réseau de distribution en Afrique.

\subsection{Fonction technique d'incubateur}

L'entreprise familiale remplit également la fonction d'incubateur surtout auprès des plus jeunes membres de la famille à qui revient la charge de prendre la relève et de pérenniser le patrimoine familial. L'incubation est un terme emprunté à la biologie. Il signifie l'action de couver un fotus, puis un petit être vivant jusqu'à ce qu'il puisse voler de ses propres ailes. La poule incube ses œufs pendant 21 jours puis ses poussins, avant de les laisser se débrouiller tout seul dans la nature. Cette période est une période d'apprentissage intense qui sert à transférer les connaissances nécessaires à la survie.

L'économie de l'entreprise et les sciences de gestion ont récupéré ce concept biologique pour traduire le rôle d'assistance que peuvent jouer certains organismes dans la création et le développement des PME. On pense même que certaines institutions de formation en management peuvent être des incubateurs pour des projets de création d'entreprise de leurs étudiants en formation. Or, si l'on observe attentivement l'expérience séculaire, la première forme d'incubation a été assurée par l'entreprise familiale. Bon nombre de PME et d'entrepreneurs qui existent aujourd'hui ont bénéficié d'une assistance étroite des structures familiales. Ce sont ces entreprises qui leur ont fourni patiemment, pendant de longues années, dès leur plus jeune âge, l'apprentissage et les compétences nécessaires dans le métier et dans la gestion, dans un double objectif: assurer la relève du père ou de la mère entrepreneur ou acquérir des compétences pouvant aider à la création et au développement de sa propre entreprise. Dans ces conditions, l'entreprise familiale devient une source de valorisation des métiers et des compétences transmis de génération en génération.

Dans une enquête récente portant sur 50 PME implantées dans la ville de Brazzaville (Boungou Bazika, 2005), nous avons constaté que 30\% des entrepreneurs ont reçu leur apprentissage de leur père dans le domaine de la soudure et celui de la menuiserie. Ces entrepreneurs, fait étonnant, ont un niveau de formation supérieur, sont des chefs d'entreprise et participent directement à la production. On peut citer l'exemple d'un jeune entrepreneur détenant une maîtrise en économie et possédant en même temps la qualification dans la menuiserie. À la question de savoir comment il s'y est pris pour avoir deux qualifications, il a répondu que, très

Revue internationale P.M.E., vol. 18, n ${ }^{\text {os }} 3-4,2005$

(C) 2005 - Presses de l'Université du Québec

Édifice Le Delta I, 2875, boul. Laurier, bureau 450, Sainte-Foy, Québec G1V 2M2 • Tél.: (418) 657-4399 - www.puq.ca

Tiré de: Revue internationale P.M.E., vol. 18, nos $3-4$, sous la direction de Louis Raymond - PME1803N

Tous droits de reproduction, de traduction et d'adaptation réservés 
tôt, son père l'avait poussé à aller le plus loin possible dans les études tout en le formant lui-même dans le métier de menuisier. Actuellement, il gère une entreprise de près de 10 salariés alors que son père possédait un ou deux apprentis.

Le rôle d'incubateur peut aussi se traduire par la transmission d'une expérience de gestion, de conduite des hommes, de maniement de fonds, etc. Une fois l'apprentissage mené à terme et les compétences assurées, le jeune membre de la famille est laissé libre de jouer un rôle de responsabilité dans l'entreprise familiale, ou alors de voler de ses propres ailes en créant sa propre entreprise.

\subsection{Fonction de financement du capital de démarrage}

L'entreprise familiale remplit aussi une autre fonction: la fonction financière. Elle consiste à apporter des financements à d'autres membres de la famille qui souhaiteraient se lancer dans les affaires. Elle peut aussi, dans certaines conditions, autofinancer ses activités de diversification des produits sur le marché.

Dans le premier cas, le financement qu'elle est en mesure d'apporter à d'autres membres de la famille procède du principe évoqué plus haut, soit celui de la coopération et de l'entraide. Il est naturel que des membres d'une même famille fassent preuve de solidarité en aidant les personnes dont les compétences peuvent conduire à la création d'entreprises viables et possédant des chances de réussite. D'ailleurs, cette aide financière est perçue comme un moyen de diversifier les activités de la famille, d'accroître sa puissance et de garantir sa pérennité dans les affaires. Un membre de la famille aidé par elle pour lancer une affaire demeure reconnaissant. Il a vis-à-vis des autres membres une dette morale et devra à son tour être solidaire pour venir en aide aux autres membres en difficulté ou aux plus jeunes. On peut donc considérer cette forme d'entraide comme une forme d'assurance contre les aléas de l'environnement. La persistance des pratiques communautaires en Afrique constitue un fondement solide à cette aide intrafamiliale. En outre, la vulnérabilité des agents à l'instabilité de l'environnement économique et social renforce cette pratique.

Enfin, l'entreprise familiale peut aussi utiliser ses surplus pour autofinancer d'autres activités. Il s'agira soit d'étendre sur le plan géographique son implantation, soit de diversifier la gamme de ses produits.

De façon générale, la fonction de financement assumée par l'entreprise familiale acquiert une grande importance dans les conditions des économies africaines marquées par des déficiences du système de crédit. En effet, il est souvent admis que les banques en Afrique ne financent pas les investissements des entreprises, le haut du bilan. Les fonds propres des PME proviennent principalement de l'épargne personnelle ou de l'aide familiale (Fauré et Labazée, 2000). Cette lacune s'explique par la nature des dépôts qui constituent les ressources des banques: ce sont des

Revue internationale P.M.E., vol. 18, ${ }^{\text {os }} 3-4,2005$

(C) 2005 - Presses de l'Université du Québec

Édifice Le Delta I, 2875, boul. Laurier, bureau 450, Sainte-Foy, Québec G1V 2M2 • Tél.: (418) 657-4399 - www.puq.ca

Tiré de: Revue internationale P.M.E., vol. 18 , $\mathrm{n}^{\text {os }} 3-4$, sous la direction de Louis Raymond $\cdot$ PME1803N

Tous droits de reproduction, de traduction et d'adaptation réservés 
dépôts à vue. Il est difficile de financer des fonds propres avec des ressources à court terme, d'autant plus que les sociétés de capital-risque sont presque inexistantes. Celles qui existent sont plus intéressées au financement du développement de l'entreprise et non au financement de sa création, phase considérée comme comportant un haut risque de mortalité pour l'entreprise (Ababacar, 2003).

En outre, l'absence d'un marché financier où peuvent se négocier les titres contribuant à assurer des financements longs pour les entreprises est un sérieux handicap au financement de celles-ci. Dans ces conditions, l'entreprise familiale, lorsqu'elle engendre d'importants excédents, représente une source de financement intéressante et indispensable pour les futurs créateurs d'entreprises issus de ses rangs.

Dans l'enquête sur les 50 PME évoquée plus haut, il a été constaté que certaines d'entre elles ont pu financer leur capital de démarrage grâce aux subventions reçues de l'entreprise dirigée par le père. Le pourcentage de ce genre d'entreprises est estimé à $25 \%$. Aucune n'a bénéficié d'un financement bancaire. Ces résultats sont corroborés par d'autres enquêtes menées en Côte-d'Ivoire, au Burkina Faso ou au Sénégal sur le financement des PME.

\subsection{Fonction de gestion des ressources humaines à faible coût}

L'entreprise familiale possède une autre fonction non moins importante dans les pays africains, soit celle de pourvoyeuse et de gestion des emplois à faible coût. Si elle ne remplissait pas cette fonction, un grand nombre de personnes n'auraient pas pu s'insérer dans le monde de l'entreprise. L'entreprise familiale permet de résorber la main-d'œuvre non utilisée, mais pas n'importe quelle main-d'œuvre, ce sont les actifs faisant partie de la famille, ce que Marniese et Morisson (2000) appellent les «aides familiaux». En effet, dans leur récente étude sur la dynamique de la microentreprise, ils montrent que la flexibilité des microentreprises provient de leur capacité à s'adapter aux chocs économiques et à l'évolution du marché caractérisé par une forte concurrence. Le facteur essentiel qui explique cette flexibilité plus grande par rapport aux autres types d'entreprises est la possibilité de baisser les coûts de production et les coûts de transaction en utilisant une maind'œuvre composée essentiellement d'aides familiaux sous-payés et même parfois non rémunérés.

En contrepartie, l'entrepreneur peut leur assurer la survie en prenant en charge leurs frais de logement, d'alimentation et de santé. Cette main-d'œuvre quasi gratuite, à l'égard de laquelle elle n'assume aucune charge sociale, contribue à l'accroissement de la compétitivité de l'entreprise.

Dans les pays africains où se pose avec acuité le problème du chômage, cette fonction revêt une importance particulière. Le personnel des entreprises familiales

Revue internationale P.M.E., vol. 18, n ${ }^{\text {os }} 3-4,2005$

(C) 2005 - Presses de l'Université du Québec

Édifice Le Delta I, 2875, boul. Laurier, bureau 450, Sainte-Foy, Québec G1V 2M2 - Tél.: (418) 657-4399 - www.puq.ca

Tiré de: Revue internationale P.M.E., vol. 18, nos $3-4$, sous la direction de Louis Raymond - PME1803N

Tous droits de reproduction, de traduction et d'adaptation réservés 
est principalement constitué des membres de la famille élargie de l'entrepreneur ou de ses amis proches. La fourniture de l'emploi représente la traduction du principe de solidarité pour l'entrepreneur et l'acceptation d'un emploi sous-payé est la contrepartie fournie par le demandeur. Il doit aider la famille à préserver et accroître le bien de tous, l'entreprise familiale, en acceptant de travailler presque gratuitement.

Barthelemy (1986) montre comment le principe de solidarité agit sur le fonctionnement de l'entreprise et lui confère ce caractère familial: «Le destin solidaire de survie autour d'un patrimoine unique contribue à rendre automatique cette intervention, souvent peu apparente bien que très réelle dans le fonctionnement de l'entreprise. C'est d'abord l'épouse qui intervient (commerce, secrétariat, permanence au téléphone, réception des clients, comptabilité), mais également les enfants ou les proches parents» (p. 13).

En France, on évaluait à 160000 le nombre des collaborateurs familiaux non rémunérés et, en 1976, selon une enquête du CREDOC, $21 \%$ des épouses consacraient de 12 à 50 heures par semaine à l'entreprise familiale. Cette situation a conduit les pouvoirs publics à reconnaître en 1982 un statut officiel du conjoint dans le commerce et l'artisanat (Barthelemy, 1986, p. 13).

Dans les pays africains où règne l'entreprise informelle, le statut du conjoint n'est pas reconnu et la contribution de la femme dans les activités de l'entreprise familiale n'est pas juridiquement prise en compte, sauf si celle-ci, dans le cadre d'une société à responsabilité limitée, a réalisé un apport en capital et, en tant qu'associée, bénéficie des droits juridiques relatifs. Mais ce sont des cas plutôt exceptionnels étant donné la domination du secteur informel.

Dans le même sens, le recrutement des apprentis est un moyen non seulement de former des jeunes, mais aussi de disposer d'une main-d'œuvre quasi gratuite. C'est le constat fait par Morice lorsqu'il étudie les travailleurs du métal dans la ville sénégalaise de Kaolack (cité dans Barthélémy, 1986, p. 14-15).

Déjà, en 1776, Smith (1976) soulignait le caractère gratuit de l'apprentissage lorsqu'il analysait dans Recherches sur la nature et les causes de la richesse des nations, les différentes formes d'emplois. «Tant que dure l'apprentissage, tout le travail de l'apprenti appartient à son maître: pendant ce même temps, il faut souvent que sa nourriture soit payée par ses père et mère ou quelque autre de ses parents et presque toujours il faut au moins qu'ils l'habillent» (p. 107).

Plusieurs auteurs soulignent l'importance du contexte familial dans le recrutement des apprentis (Leonard, 1980, Devauges, 1979, etc. ${ }^{6}$.

6. Cité par Barthélémy, p. 16.

Revue internationale P.M.E., vol. 18, ${ }^{\text {os }} 3-4,2005$

(C) 2005 - Presses de l'Université du Québec

Édifice Le Delta I, 2875, boul. Laurier, bureau 450, Sainte-Foy, Québec G1V 2M2 • Tél.: (418) 657-4399 - www.puq.ca

Tiré de: Revue internationale P.M.E., vol. 18, nos 3-4, sous la direction de Louis Raymond • PME1803N

Tous droits de reproduction, de traduction et d'adaptation réservés 
Il est distingué trois classes d'apprentis:

- les successeurs potentiels au patron, destinés à aider celui-ci dans ses vieux jours;

- la réserve de main-d'œuvre permanente de l'atelier, composée d'apprentis plus ou moins qualifiés et plus ou moins utilisés, selon l'urgence du travail;

- la main-d'œuvre occasionnelle. L'état d'apprenti dans ces conditions s'exerce hors de tout statut, sans obligation réelle de la part du patron et avec un revenu insuffisant et irrégulier (Barthélémy, 1986).

Ces apprentis sont les cousins ou neveux de l'entrepreneur. Il doit les former, mais, en même temps, les utiliser comme main-d'œuvre d'appoint venant concurrencer les ouvriers, car susceptible de les remplacer à un moment ou un autre. Le déclin de la grande famille africaine annonce le déclin de l'entreprise familiale.

\section{Tableau 1}

Typologie des entreprises familiales et individuelles

\begin{tabular}{lll}
\hline Caractéristiques & $\begin{array}{l}\text { Entreprises } \\
\text { individuelles }\end{array}$ & Entreprises familiales \\
\hline Principe de base & individualisme & solidarité \\
Gestion & personnelle & collective \\
Ressources & hiérargne personnelle & fonctionnelle \\
Organisation & par le propriétaire seul & par consensus \\
Prise de décision & dépend de la vie & dépend de la cohésion \\
Pérennité & du propriétaire & familiale \\
& grande & limitée \\
Possibilité de croissance & rapide & lent \\
Temps de décision & développement & déclin \\
Perspective & secrète & transparente \\
Culture de gestion & &
\end{tabular}

Cette typologie est une schématisation de la réalité, cette dernière étant souvent bien plus complexe. Il est évident que la typologie énoncée n'intègre pas les formes intermédiaires d'entreprises, celles se situant à mi-chemin entre les deux modèles. Il est possible de distinguer au moins trois formes intermédiaires: l'entreprise créée par un individu, qui après avoir prospéré est devenue, en raison du processus successoral, un patrimoine familial; l'entreprise familiale par les capitaux mais gouvernée comme une entreprise individuelle parce que les cadets, fournisseurs de capitaux, doivent une obéissance absolue à l'aîné, le dirigeant de droit et de fait de l'entreprise; l'entreprise individuelle par les capitaux, mais insérée dans le contexte familial par les ressources humaines, celle-ci étant alors gérée de façon collective. 


\subsection{Limites}

Nous venons d'esquisser la définition de l'entreprise familiale ainsi que ses différentes fonctions. Nous avons indiqué les difficultés méthodologiques qu'il faut surmonter pour définir l'entreprise familiale, particulièrement en l'inscrivant dans une perspective africaine. Il nous semble important de souligner les limites de l'entreprise familiale, notamment deux limites essentielles.

1. L'entreprise familiale a du mal à s'adapter à l'évolution des pays africains marquée par une rapide et forte urbanisation. La ville est de plus en plus composée de citadins stratifiés en fonction du lieu où ils sont nés et ont grandi. Les citadins de la première génération sont nettement portés vers la solidarité familiale tandis que ceux de la deuxième et troisième génération sont plus marqués par l'individualisme. Ils remettent en cause le mode de vie communautaire, ce qui tend à désagréger la cellule familiale élargie. Cette tendance découle de la localisation qui ne dépend plus de la cellule familiale ou de la communauté ethnique, mais des revenus du ménage. Si, dans les années 1960, la famille avait tendance à vivre ensemble, aujourd'hui, avec l'urbanisation, la monétarisation et la crise économique, les couples tendent à s'individualiser et à s'isoler en louant un appartement loin de la cellule familiale d'origine. Cette dispersion renforce l'individualisme et crée des conditions qui favorisent l'implantation d'entreprises individuelles au détriment d'entreprises familiales. Par ailleurs, l'émergence de divers modèles familiaux (familles monoparentales, femmes chefs de famille, personnes isolées, etc.) tend à renforcer le processus d'invidualisation en zones urbaines et la constitution d'entreprises individuelles plutôt que familiales.

2. L'entreprise familiale est une contrainte pour le jeune qui veut s'émanciper de la tutelle familiale et des charges qu'elle engendre: charges en dépenses de consommation pour un nombre élargi de personnes, charges en règlement de multiples conflits qui naissent au sein de la famille, etc. La crise économique et sociale que traversent les pays africains tend à aggraver les difficultés des ménages et à limiter les possibilités concrètes d'entraide (Marie, 1997). Des disparités importantes surgissent au sein de la famille entre les nantis et les démunis, les lettrés et les illettrés, les ruraux et les citadins, les garçons et les filles, les aînés et les cadets. Erny (1987) montrait déjà que la famille en Afrique était loin d'être une entité homogène. Elle est caractérisée par la coexistence d'individus disparates en termes de revenus, niveau d'instruction, religion, etc. Ces disparités conduisent à des perceptions et des modes de vie différents et même contradictoires, engendrent toutes sortes de conflits et accentuent l'individualisation des membres d'une même famille. Dans ce contexte,

Revue internationale P.M.E., vol. 18, $\mathrm{n}^{\text {os }} 3-4,2005$

(C) 2005 - Presses de l'Université du Québec

Édifice Le Delta I, 2875, boul. Laurier, bureau 450, Sainte-Foy, Québec G1V 2M2 • Tél.: (418) 657-4399 - www.puq.ca

Tiré de: Revue internationale P.M.E., vol. 18, nos $3-4$, sous la direction de Louis Raymond • PME1803N

Tous droits de reproduction, de traduction et d'adaptation réservés 
de plus en plus d'entrepreneurs en Afrique tendent à constituer des entreprises individuelles. Ils vont même parfois jusqu'à cacher l'existence de leur patrimoine à leur famille afin de réduire les charges qui pourront peser sur les marges de ces unités de production (nécessité de recruter des membres de la famille même quand cela n'est pas utile, de subvenir à leurs besoins au risque de compromettre la rentabilité de l'affaire, etc.) ainsi que les sources d'antagonisme. En outre, les conceptions magicomystiques tendraient à faire croire que le membre de la famille qui aurait réussi pourrait être victime de malédiction d'un de ses parents s'il ne lui consent pas une part des excédents générés par ses affaires. Toutes ces raisons objectives et subjectives entraînent la création d'un nombre croissant d'entreprises individuelles plutôt que d'entreprises familiales dans les pays africains, tout en renforçant la culture du secret dans les affaires.

On pourrait s'acheminer alors vers une individualisation croissante des affaires, individualisation qui tend à se renforcer en même temps que la famille est traversée par une crise de grande ampleur qui remet aujourd'hui en cause non seulement sa configuration élargie au profit du noyau conjugal, mais aussi ses fondements marqués par les principes traditionnels d'entraide et de collaboration. Par ailleurs, le partenariat entre deux familles de race différente constitue un progrès et un enrichissement en management pour les entreprises familiales africaines, car il permet de limiter l'influence du social sur l'économique et de garantir des règles de gestion modernes et plus rigoureuses.

\section{Conclusion}

L'entreprise familiale est une entité qui pose problème dans son appréhension théorique et son contour empirique. Le rôle historique qu'elle joue est indéniable; elle continue d'être le creuset où se forgent bon nombre d'entrepreneurs africains. Cependant, l'urbanisation et l'individualisation croissante et, parallèlement, le déclin de la famille élargie au profit de la famille nucléaire tendent à limiter l'expansion de l'entreprise familiale dans les pays africains au profit de l'entreprise individuelle. Naturellement, cette évolution vers l'individualisation de l'entrepreneuriat se situe à des degrés divers, car le processus de nucléarisation de la famille comporte des particularités propres à chaque pays en Afrique. Certains pays sont très urbanisés et le poids de la famille nucléaire s'y accentue. C'est le cas du Congo où $57 \%$ de la population vit dans les villes. D'autres, par contre, ont encore une importante population rurale; c'est le cas du Cameroun, du Burkina, du Mali, etc.

Une des pistes de recherche pourrait consister à étudier certaines entreprises familiales qui ont réussi ou connu des échecs et d'expliquer les facteurs ayant

Revue internationale P.M.E., vol. 18, $\mathrm{n}^{\text {os }} 3-4,2005$

(C) 2005 - Presses de l'Université du Québec

Édifice Le Delta I, 2875, boul. Laurier, bureau 450, Sainte-Foy, Québec G1V 2M2 - Tél.: (418) 657-4399 - www.puq.ca

Tiré de: Revue internationale P.M.E., vol. 18, nos 3-4, sous la direction de Louis Raymond • PME1803N

Tous droits de reproduction, de traduction et d'adaptation réservés 
déterminé ces résultats. De même, de larges enquêtes pourraient être réalisées afin de mieux cerner l'ampleur réelle des entreprises familiales, les points de similitude et de différenciation des entreprises familiales.

\section{Bibliographie}

AbabacAR, D.S. (2003), «Bilan analytique des activités de capital risque en Afrique subsaharienne», Actes des VIII ${ }^{\text {es }}$ Journées scientifiques du réseau Entrepreneuriat de l'Agence universitaire pour la francophonie, 21-24 mai, Rouen.

Barthélémy, G. (1986), Artisanat et développement, Paris, CRET.

BAuman, E. (2003), «La solidarité autrement? Micro-assurance et protection contre la vulnérabilité en Afrique de l'Ouest», Actes des VIII ${ }^{\text {es }}$ Journées scientifiques du réseau Entrepreneuriat de l'Agence universitaire pour la francophonie, 21-24 mai, Rouen.

Bertrand, M. (2003): «Familles urbaines du Ghana: circulation des cadets, positions résidentielles des aînés», Ves Journées scientifiques du réseau Démographie de l'AUF, 23-26 juin, Marseille.

Boungou BaziKa, J.-C. (2005), Entrepreneuriat et innovation au Congo-Brazzaville, à paraître.

Boungou BAZIKA, J.-C. et R. SAmBa (2002), «Le commerce informel et l'agriculture périurbaine: deux secteurs d'activité des jeunes touchés par la pauvreté en République du Congo», Rapport d'étude de l'Université M.-Ngouabi-PNUD, Brazzaville.

Bousseta, M. (2003), «Contraintes technologiques, innovations et compétitivité des petites et moyennes entreprises au Maroc: résultats d'une étude empirique», Actes des VIII ${ }^{\text {es }}$ Journées scientifiques du réseau Entrepreneuriat de l'Agence universitaire pour la francophonie, 21-24 mai, Rouen.

Connu, J.-F. (1998), «La reconquête d'une filière», Groupe de l'AFD (dir.), L'Afrique des entreprises, Paris, La Documentation française, p. 247-258.

ERny, P. (1987), L'enfant et son milieu en Afrique noire, Paris, L'Harmattan.

FAuré, Y.-A. et P. LABAZÉE (dir.) (2000), Petits patrons africains: entre l'assistance et le marché, Paris, Karthala, 644 p.

Guitton, H. et D. Vitry (1991), Économie politique, Paris, Dalloz, 819 p.

Kodjopatapa, L.A. (2003), «Étude de l'évolution des familles au Togo dans un contexte de crise économique», V's Journées scientifiques du réseau Démographie de l'AUF, 23-26 juin, Marseille.

Kombou, L. (2002), «Les PME camerounaises et l'innovation», Revue africaine d'économie et de gestion, $\mathrm{n}^{\circ}$ 8, p. 21-35.

Hernandez, E.-M. (2002), «De nouvelles règles pour entreprendre», Problèmes économiques, $\mathrm{n}^{\circ} 2.764,5$ juin, p. 29-32.

Hugon, P. (1995), «Les entrepreneurs africains et l'analyse économique», dans S. Ellis et A. Fauré (dir.), Entreprises et entrepreneurs africains, Paris, Karthala, chapitre 29, $632 \mathrm{p}$.

Revue internationale P.M.E., vol. 18, ${ }^{\text {os }} 3-4,2005$

(C) 2005 - Presses de l'Université du Québec

Édifice Le Delta I, 2875, boul. Laurier, bureau 450, Sainte-Foy, Québec G1V 2M2 • Tél.: (418) 657-4399 - www.puq.ca

Tiré de: Revue internationale P.M.E., vol. 18, nos 3-4, sous la direction de Louis Raymond • PME1803N

Tous droits de reproduction, de traduction et d'adaptation réservés 
MARIE, A. (1997), «Les structures familiales à l'épreuve de l'individualisation citadine», dans M. Pilon, T. Locoh, E. Vignikin et P. Vimard (dir.), Ménages et familles en Afrique, approches des dynamiques contemporaines, Paris, CPD, chapitre 14.

Marniesse, S. et C. Morrisson (2000), «Dynamique des micro-entreprises, de nouveaux enseignements », Revue d'économie du développement, $\mathrm{n}^{\circ}$ 4, décembre, p. 3-33.

Penoull, M. (1979), Socio-économie du sous-développement, Paris, Dalloz.

Sмітн, A. (1976), Recherches sur la nature et les causes de la richesse des nations, Paris, Gallimard, $445 \mathrm{p}$.

Soulié, D. (1992), Analyse économique et stratégie d'entreprise, Vanves, Éditions Edicef, $285 \mathrm{p}$.

Torrès, O. (2001), «Les divers types d'entrepreneuriat et de PME dans le monde», Revue du management international, vol. $6, \mathrm{n}^{\circ} 1$, p. 1-15.

Vimard, P. et S. N'Cho (1997), «Évolution de la structure des ménages et différenciation des modèles familiaux en Côte d'Ivoire, 1975-1993», dans M. Pilon, T. Locoh, E. Vignikin et P. Vimard (dir.), Ménages et familles en Afrique, approches des dynamiques contemporaines, Paris, CPD, chapitre 5.

WAKAM, J. (1997), «Différenciation socio-économique et structures familiales au Cameroun», dans M. Pilon, T. Locoh, E. Vignikin et P. Vimard (dir.), Ménages et familles en Afrique, approches des dynamiques contemporaines, Paris, CPD, chapitre 13.

Revue internationale P.M.E., vol. 18, nos 3-4, 2005

(c) 2005 - Presses de l'Université du Québec

Édifice Le Delta I, 2875, boul. Laurier, bureau 450, Sainte-Foy, Québec G1V 2M2 - Tél.: (418) 657-4399 - www.puq.ca

Tiré de: Revue internationale P.M.E., vol. 18, nos $3-4$, sous la direction de Louis Raymond - PME1803N

Tous droits de reproduction, de traduction et d'adaptation réservés 